\title{
Every Banach space is reflexive
}

\author{
Christophe Van Olmen and Stijn Verwulgen
}

\begin{abstract}
The title above is wrong, because the strong dual of a Banach space is too strong to assert that the natural correspondence between a space and its bidual is an isomorphism. This, from a categorical point of view, is indeed the right duality concept because it yields a self adjoint dualisation functor. However, for many applications the non-reflexiveness problem can be solved by replacing the norm on the first dual by the weak*-structure [1].

But then, by taking the second dual, only the original vector space is recovered and no universal property remains with this modified dual structure. In this work we unify the applied and the structural point of view.

We introduce a suitable numerical structure on vector spaces such that Banach balls, or more precisely totally convex modules, arise naturally in duality, i.e. as a category of Eilenberg-Moore algebras. This numerical structure naturally overlies the weak*-topology on the algebraic dual, so the entire Banach space can be reconstructed as a second dual. Moreover, the isomorphism between the original space and its bidual is the unit of an adjunction between the two dualisation functors.

Notice that the weak*-topology is normable only if it lives on a finite dimensional space; in that case the original space is trivial as well, hence reflexive. So the overlying numerical structure should be something more general than a norm or a seminorm and thus approach theory $[2,3]$ enters the picture.
\end{abstract}

AMS Subject Classification: 18C20, 46B10, 52A01.

Keywords: locally convex approach space, duality, totally convex module, Banach space, weak* structure

\section{Introduction and preliminaries}

For a set $S$, let $l_{1} S$ denote the space $\{a: S \rightarrow \mathbb{R} \mid\{a \neq 0\}$ is countable $\}$ equipped with the sum-norm $\|a\|_{1}:=\sum_{s \in S}|a(s)|$. The assignment $S \longmapsto l_{1} S$ gives rise to a functor

$$
l_{1}: \text { Set } \rightarrow \operatorname{Ban}_{1}:\left(S_{1} \stackrel{f}{\rightarrow} S_{2}\right) \longmapsto\left(l_{1} S_{1} \stackrel{l_{1} f}{\longrightarrow} l_{1} S_{2}\right),
$$

with $l_{1} f(a):=\sum_{s \in S_{1}} a(s) \delta_{f(s)}$, which is left adjoint to the closed unit ball functor

$$
O: \mathbf{B a n}_{1} \rightarrow \text { Set }: X \stackrel{f}{\rightarrow} Y \longmapsto O X \stackrel{\left.f\right|_{O X X}}{\rightarrow} O Y .
$$

We put $\mathbf{B a n}_{1}$ for the category of Banach spaces and linear non-expansive maps. A totally convex module is the abstraction of the algebraic structure on the closed unit ball of a Banach space, that is, the category $\mathbf{T C}$ of totally convex modules and totally convex maps arises as the Eilenberg-Moore algebras induced by the adjunction $l_{1} \dashv O$.

A totally convex module was characterized in [6] as a set $X$ on which, for each sequence of scalars $\alpha=\left(\alpha_{n}\right)_{n \in \mathbb{N}}$, with $\sum_{n \in \mathbb{N}}\left|\alpha_{n}\right| \leq 1$, an operation $\widehat{\alpha}: X^{\mathbb{N}} \rightarrow X$ is given, such that, with $\sum_{n \in \mathbb{N}}^{n} \alpha_{n} x_{n}:=$ $\widehat{\alpha}\left(\left(x_{n}\right)_{n}\right)$, the following identities are satisfied:

(TC1) $\sum_{n \in \mathbb{N}} \delta_{n}(k) x_{n}=x_{k}$

$(\mathrm{TC} 2) \sum_{j \in \mathbb{N}} \beta_{j}\left(\sum_{n \in \mathbb{N}} \alpha_{n j} x_{n}\right)=\sum_{n \in \mathbb{N}}\left(\sum_{j \in \mathbb{N}} \beta_{j} \alpha_{n j}\right) x_{n} ;$ 
a map between totally convex modules is called totally convex if it preserves these formal totally convex combinations. The pointwise totally convex structure on the closed unit ball of a seminormed space $X$ is denoted $\widehat{O} X$. This induces the comparison functor $\widehat{O}: \mathbf{s N o r m}_{1} \rightarrow \mathbf{T C}$ of the adjunction $l_{1} \dashv O$. A similar algebraic structure is obtained if the above algebraic laws are imposed to summation operations associated to sequences of finite length (for which the sum of the absolute values does not exceed 1). These structures are called absolutely convex modules and, together with absolutely affine maps, they form the category AC. It can be seen that we have a full embedding $\mathbf{T C} \hookrightarrow \mathbf{A C}[6]$. Totally convex and absolutely convex modules were first mentioned by Semadeni [11], then they were clearly defined and intensively studied by Pumplün and Röhrl $[6,7,8,9,10,5]$.

An approach vector space is a vector space together with an approach structure that concords well with the algebraic structure of the space [4]. We recollect some facts about locally convex approach spaces (those approach vector spaces satisfying a certain local convexity condition) [14]. First of all, a locally convex approach space was characterized as a pair $X=(X, \mathcal{M})$, consisting of a vector space $X$ equipped with a Minkowski system $\mathcal{M}$. The latter is an ideal in the lattice of seminorms on $X, \operatorname{Sn}(X)$, satisfying the following saturation condition:

(S) $\forall \eta \in \operatorname{Sn}(X), \forall \epsilon>0 \exists \mu \in \mathcal{M}: \eta \leq(1+\epsilon) \mu \Rightarrow \eta \in \mathcal{M}$.

The saturation of an ideal basis (the smallest subset of $\operatorname{Sn}(X)$ containing that collection and satisfying $(\mathrm{S})$ ) is a Minkowski system. A morphism between locally convex approach spaces is a map that is linear and a contraction with respect to the approach structures. This means that a linear map $f:\left(X, \mathcal{M}_{X}\right) \rightarrow\left(Y, \mathcal{M}_{Y}\right)$ is a morphism if for all $\eta \in \mathcal{M}_{Y}: \eta \circ f \in \mathcal{M}_{X}$. We put lcApVec for the category of locally convex approach spaces. Now, any locally convex topology is uniquely determined by the collection of Minkowski functionals of absolutely convex zero-neighborhoods. So, it is easy to see that locally convex approach spaces are a generalization of locally convex topological spaces. Moreover, it was shown that the category lcApVec is topological over Vec and that limits and colimits in lcTopVec are unaltered under the presence of this supercategory. The coreflection corresponding to the right adjoint of the embedding lcTopVec $\hookrightarrow \mathbf{l c A p V e c}$ is given by the identity $\operatorname{map}\left(X, \mathbb{R}^{+} \mathcal{M}\right) \longrightarrow(X, \mathcal{M})$, with $\mathbb{R}^{+} \mathcal{M}:=\{k \eta \mid k>0, \eta \in \mathcal{M}\}$. Note that the corresponding topology is precisely the topology underlying $\mathcal{M}$. Thus, if the classical quantitative theory is also nicely incorporated, we have a rigid framework for doing quantified functional analysis. This is indeed the case because for a seminormed space $(X, \eta)$ we can put $\mathcal{M}_{\eta}:=\{\mu \in \operatorname{Sn}(X) \mid \mu \leq \eta\}$ and thus, under the identification $\eta \equiv \mathcal{M}_{\eta}$, we obtain a full embedding $\mathbf{s N o r m}_{1} \hookrightarrow \mathbf{l c A p V e c}$. In particular, $(\mathbb{R},||)$ is considered to be a locally convex approach space. We have a right adjoint $N: \mathbf{l c} \mathbf{A p V e c} \longrightarrow \mathbf{s N o r m}_{1}$ to this embedding: the coreflection arrow, on a locally convex approach space $(X, \mathcal{M})$, is given by the injection $N(X, \mathcal{M}) \hookrightarrow(X, \mathcal{M})$, where $N(X, \mathcal{M})$ denotes the space $\left\{x \in X \mid \sup _{\mu \in \mathcal{M}} \mu(x)<\infty\right\}$, equipped with the seminorm $x \longmapsto \sup _{\mu \in \mathcal{M}} \mu(x)$.

There is only a pointwise vector space structure on the dual of a locally convex topological space. But the dual of a quantified space has a richer structure: the homset $K X:=[X, \mathbb{R}]$ of a locally convex approach space $X$ is the closed unit ball of a seminorm on the space of all linear continuous functionals on $X$; the resulting seminormed space is denoted $L_{\widehat{K}} X$ [15]. There is also a converse connection ([15], 2.11): if we start with a vector space $X$ and closed unit ball $X^{\odot}$ in the algebraic dual of $X$ and endow $X$ with $\mathcal{M}_{(X, X \odot)}$, the inital lcApVec structure of the source $(X \stackrel{\varphi}{\rightarrow} \mathbb{R})_{\varphi \in X \odot}$, then

$$
K\left(X, \mathcal{M}_{(X, X \odot)}\right)=X^{\odot} .
$$

This nice duality is enriched with the surprising result that the category of absolutely convex modules is obtained as the category of Eilenberg-Moore algebras induced by the natural dualisation functor $K:=[-, \mathbb{R}]: \mathbf{l} \mathbf{c} \mathbf{A p V e c}{ }^{\text {op }} \rightarrow$ Set, with the pointwise absolutely convex structure of linear contractions defining the comparison functor $\widehat{K}[12]$.

Another core observation is the fact that the corresponding topological dualisation functor

$$
L_{\widehat{K}}: \mathbf{l c A p V e c}^{\mathrm{op}} \rightarrow \mathbf{s N o r m}_{1}:(X \stackrel{f}{\rightarrow} Y) \longmapsto\left(L_{\widehat{K}} Y \stackrel{L_{\widehat{K}}}{\longrightarrow} L_{\widehat{K}} X: a \longmapsto a \circ f\right)
$$

has a left adjoint

$$
L_{\mathrm{pcu}}: \mathbf{s N o r m}_{1} \longrightarrow \mathbf{l c A p V e c}{ }^{\mathrm{op}}:(X \stackrel{f}{\rightarrow} Y) \longmapsto\left(L_{\mathrm{pcu}} Y \stackrel{L_{\mathrm{pcu}} f}{\rightarrow} L_{\mathrm{pcu}} X: a \longmapsto a \circ f\right)
$$


[12]. Here $L_{\mathrm{pcu}} X$ is the algebraic dual of a seminormed space $X$ equipped with the saturation of basis $\left\{\sup _{x \in S}|\operatorname{ev}(-, x)| \mid S \subset O X\right.$ finite $\}$. The subscript pcu-pointwise convergence on the unit ball-is explained by the fact that the underlying qualitative structure is the weak* topology.

It is interesting to see that the categorical relations between the qualitative and the quantitative level of functional analysis are transported by dualisation to the embedding Vec $\hookrightarrow$ AC [16]. The question arises whether we can also obtain the subcategory $\mathbf{T C} \hookrightarrow \mathbf{A C}$ via dualisation, as the Eilenberg-Moore category of a suitable subcategory of lcApVec. We will demonstrate, not only that the answer is affirmative, but also that, when $L_{\widehat{K}}$ is restricted to this subcategory, we obtain a dual adjunction with $\operatorname{Ban}_{1}$.

Note that, although the dual of a seminormed space is a Banach space, natural algebraic structure of linear contractive functionals on a seminormed space is of strictly stronger nature than the totally convex structure of this Banach ball [13]. So the subcategory $\mathbf{s N o r m}_{1} \hookrightarrow \mathbf{l c A p V e c}$ is not suitable for our purpose.

We should add that all preliminary results are shown to hold for vector spaces that are defined over the field of real numbers. So in the sequel of this work we will also confine ourselves to real valued vector spaces.

\section{Dually complete spaces}

The following definition is entailed by the above objectives.

A locally convex approach space $X$ is called dually complete if $K X$ is closed under the formation of pointwise totally convex combinations. This means explicitly that, for any sequence $\left(\varphi_{n}\right)_{n \in \mathbb{N}}$ in $K X$ and for any $\left(\alpha_{n}\right)_{n \in \mathbb{N}} \in O l_{1} \mathbb{N}$, the series $\sum_{n \in \mathbb{N}} \alpha_{n} \varphi_{n}(x)$ is commutatively convergent and that the assignment $x \longmapsto \sum_{n \in \mathbb{N}} \alpha_{n} \varphi_{n}(x)$ defines a contraction on $X$. The full subcategory of lcApVec whose objects are dually complete spaces is denoted dcApVec. We put $D: \mathbf{d c A p V e c}^{\text {op }} \rightarrow$ Set for the restriction of the natural dualisation functor $K$.

If a locally convex topological space is dually complete, then the set of continuous functionals is in a pointwise way at the same time a vector space and a totally convex module. It then follows, from [6] (6.9), that the dual space is trivial. The Hahn-Banach theorem then asserts that the only continuous seminorm is the one that is identically zero. So only trivial quantitative structures can satisfy the above definition, i.e. we have introduced an intrinsic numerical concept.

Note that the seminorm coreflection $N X$ is a dually complete space, so the collection

$$
\mathcal{A}_{X}:=\{Y \in \mid \mathbf{d c A p V e c} \| Y \text { is a sub vector space of } X \text { and } Y \hookrightarrow X \text { is contractive }\}
$$

is non-empty. Let $C X$ be the space vct $\left(\cup \mathcal{A}_{X}\right)$ equipped with the final lcApVec-structure for the sink

$$
\left(Y \hookrightarrow \operatorname{vct}\left(\bigcup \mathcal{A}_{X}\right)\right)_{Y \in \mathcal{A}_{X}}
$$

Theorem 2.1. The inclusion $i_{X}: C X \hookrightarrow X$ is co-universal with respect to the embedding $\mathbf{d c A p V e c} \hookrightarrow$ lcApVec. The corresponding right adjoint $C: \mathbf{l c A p V e c} \rightarrow \mathbf{d c A p V e c}$ to the inclusion $\mathbf{d c A p V e c} \hookrightarrow$ lcApVec acts on morphisms as a restriction in domain and co-domain.

Proof. In order to show that $C X$ is dually complete, let $\left(\varphi_{n}\right)_{n \in \mathbb{N}}$ be a sequence in $K C X$ and let $\left(\alpha_{n}\right)_{n \in \mathbb{N}} \in O l_{1} \mathbb{N}$. As a composition $Y \hookrightarrow C X \stackrel{\varphi_{n}}{\rightarrow} \mathbb{R}$, the restriction of each $\varphi_{n}$ to any $Y$ in $\mathcal{A}_{X}$ is a linear contraction, so the map $\varphi_{Y}: x \longmapsto \sum_{n \in \mathbb{N}} \alpha_{n} \varphi_{n}(x): Y \rightarrow \mathbb{R}$ is a well defined linear contraction. Moreover, we have the property

$$
\left.\varphi_{Y_{1}}\right|_{Y_{1} \cap Y_{2}}=\left.\varphi_{Y_{2}}\right|_{Y_{1} \cap Y_{2}}
$$

for the collection of mapping $\left(\varphi_{Y}\right)_{Y \in \mathcal{A}_{X}}$. So there exists a unique linear map $\varphi$ : vct $\left(\bigcup \mathcal{A}_{X}\right) \rightarrow \mathbb{R}$ such that, for all $Y \in \mathcal{A}_{X},\left.\varphi\right|_{Y}=\varphi_{Y}$. The sink (3) is final, so $\varphi$ is a linear contraction. We still have to show that $\varphi$ is obtained as the pointwise totally convex combination $\sum_{n \in \mathbb{N}} \alpha_{n} \varphi_{n}$. To this end, fix $x \in C X$. 
Then $x=\sum_{i=1}^{n} x_{i}$ for some $x_{1} \in Y_{1}, \ldots, x_{n} \in Y_{n}$, each $Y_{i} \in \mathcal{A}_{X}$. So we obtain

$$
\begin{array}{rlc}
\sum_{n \in \mathbb{N}} \alpha_{n} \varphi_{n}(x) & = & \sum_{n \in \mathbb{N}} \alpha_{n} \varphi_{n}\left(\sum_{i=1}^{m} x_{i}\right) \\
& = & \sum_{i=1}^{m} \sum_{n \in \mathbb{N}} \alpha_{n} \varphi_{n}\left(x_{i}\right) \\
& = & \sum_{i=1}^{m} \varphi_{Y_{i}}\left(x_{i}\right) \\
& = & \sum_{i=1}^{m} \varphi\left(x_{i}\right) \\
= & \varphi\left(\sum_{i=1}^{m}\left(x_{i}\right)\right)
\end{array}
$$

It is easy to see that $i_{X}$ is a morphism in lcApVec. To show the co-universal property of $i_{X}$, let $Y$ be a dually complete space, let $f: Y \rightarrow X$ be a linear contraction and let $f(Y)$ be equipped with the final lcApVec-structure of the sink $Y \stackrel{f}{\rightarrow} f(Y)$. Then the injection $f(Y) \hookrightarrow X$ is a linear contraction, so $f(Y)$ is in $\mathcal{A}_{X}$. Because of the factorization

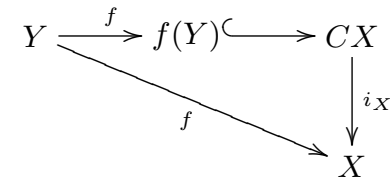

we are done if we show that $f(Y)$ is dually complete. Take $\left(\alpha_{n}\right)_{n \in \mathbb{N}} \in O l_{1} \mathbb{N}$ and a sequence $\left(\varphi_{n}\right)_{n \in \mathbb{N}}$ in $K f(Y)$. For any $f(y) \in f(Y)$ we have $\sum_{n \in \mathbb{N}} \alpha_{n} \varphi_{n}(f(y))=\sum_{n \in \mathbb{N}} \alpha_{n}\left(\varphi_{n} \circ f\right)(y)$, so the map $\sum_{n \in \mathbb{N}} \alpha_{n} \varphi_{n}: f(Y) \rightarrow \mathbb{R}$ is well-defined. Since $f: Y \rightarrow f(Y)$ is final and since $\left(\sum_{n \in \mathbb{N}} \alpha_{n} \varphi_{n}\right) \circ f$ is a linear contraction we also have that $\sum_{n \in \mathbb{N}} \alpha_{n} \varphi_{n}$ is contractive.

It follows from composition of the adjunctions in the square

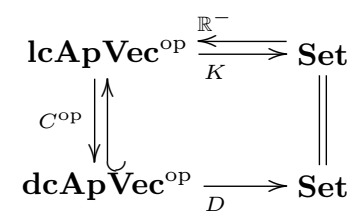

that $C^{\text {op }} \mathbb{R}^{-}:$Set $\rightarrow \mathbf{d c A p V e c}{ }^{\text {op }}$ is left adjoint to $D$. Also note that, if $f: X \rightarrow Y$ is a morphism between dually complete spaces, then $D f: \widehat{D} Y \rightarrow \widehat{D} X$ is a totally affine map.

Theorem 2.2. The category TC is a representation for the category of Eilenberg-Moore algebras induced by the adjunction $C^{\text {op }} \mathbb{R}^{-} \dashv D$ and $\widehat{D}: \mathbf{d c A p V e c}{ }^{\text {op }} \rightarrow \mathbf{T C}$ is the comparison functor of $D$.

Proof. We start with the establishment of the crucial fact that, for any set $S$, the map

$$
\tau_{S}: O l_{1} S \rightarrow D C \mathbb{R}^{S}: b \longmapsto \sum_{s \in S} b(s) \operatorname{ev}(-, s)
$$

is a well defined bijection.

For each $s \in S$, if we compose the map ev $(-, s): \mathbb{R}^{S} \rightarrow \mathbb{R}$ with the inclusion $i_{C \mathbb{R}^{S}}: C \mathbb{R}^{S} \hookrightarrow \mathbb{R}^{S}$, we obtain a contraction ev $(-, s): C \mathbb{R}^{S} \rightarrow \mathbb{R}$, so the assertion that $\tau_{S}$ is well-defined follows from the fact that $K C \mathbb{R}^{S}$ is closed under the formation of pointwise totally convex combinations.

To show that $\tau_{S}$ is an injection, take $b_{1}$ and $b_{2}$ in $O l_{1} S$ such that $b_{1}(t) \neq b_{2}(t)$ for some $t \in S$. The seminorm coreflection $N \mathbb{R}^{S}$ consists of all bounded functions from $S$ to $\mathbb{R}$ and is contained in $C \mathbb{R}^{S}$. In particular, the Dirac function $\delta_{t}$ is in $C \mathbb{R}^{S}$. So $\tau_{S}\left(b_{1}\right)\left(\delta_{t}\right)=\sum_{s \in S} b_{1}(s) \operatorname{ev}(-, s)\left(\delta_{t}\right)=b_{1}(t) \neq b_{2}(t)=$ $\tau_{S}\left(b_{2}\right)\left(\delta_{t}\right)$, i.e. $\tau_{S}\left(b_{1}\right) \neq \tau_{S}\left(b_{2}\right)$.

To conclude that $\tau_{S}$ is a surjection, we will show the inclusion $K C \mathbb{R}^{S} \subset \tau_{S}\left(O l_{1} S\right)$. Because $C \mathbb{R}^{S}$ is dually complete, $\tau_{S}\left(O l_{1} S\right)$ is, in a pointwise way, a totally convex subset of the algebraic dual of $C \mathbb{R}^{S}$. Since $\tau_{S}$ is an injection, the map $\tau_{S}: \widehat{O} l_{1} S \rightarrow \tau_{S}\left(O l_{1} S\right)$ is an isomorphism of totally convex modules. It follow from section 7 of [6] that the space $\operatorname{vct}\left(\tau_{S}\left(O l_{1} S\right)\right)$, with the Minkowski functional $\mu_{\tau_{S}\left(O l_{1} S\right)}$, is a Banach space that is universal for $\widehat{O}$ for the module $\tau_{S}\left(O l_{1} S\right)$. So the totally convex 
isomorphism $\tau_{S}: \widehat{O} l_{1} S \rightarrow \tau_{S}\left(O l_{1} S\right)$ extends to an isomorphism $\widetilde{\tau_{S}}: l_{1} S \rightarrow\left(\operatorname{vct}\left(\tau_{S}\left(O l_{1} S\right)\right), \mu_{\tau_{S}}\left(O l_{1} S\right)\right)$ between the respective free Banach spaces. In particular, $\widetilde{\tau_{S}}$ is onto on the closed unit balls, so we have $\tau_{S}\left(O l_{1} S\right)=\widetilde{\tau_{S}}\left(O l_{1} S\right)=\left\{\mu_{\tau_{S}\left(O l_{1} S\right)} \leq 1\right\}$. So from (1) we obtain the identity

$$
K\left(C \mathbb{R}^{S}, \mathcal{M}_{\left(C \mathbb{R}^{S}, \tau_{S}\left(O l_{1} S\right)\right)}\right)=\tau_{S}\left(O l_{1} S\right) .
$$

Since $\tau_{S}\left(\mathrm{Ol}_{1} S\right)$ is a totally convex module, $\left(C \mathbb{R}^{S}, \mathcal{M}_{\left(C \mathbb{R}^{S}, \tau_{S}\left(\mathrm{Ol}_{1} S\right)\right)}\right)$ is a dually complete space. Because each ev $(-, s)$ is in $\mathcal{M}_{\left(C \mathbb{R}^{S}, \tau_{S}\left(O l_{1} S\right)\right)}$, the injection $\left(C \mathbb{R}^{S}, \mathcal{M}_{\left(C \mathbb{R}^{S}, \tau_{S}\left(O l_{1} S\right)\right)}\right) \hookrightarrow \mathbb{R}^{S}$ is contractive. So we have that $\left(C \mathbb{R}^{S}, \mathcal{M}_{\left(C \mathbb{R}^{S}, \tau_{S}\left(O l_{1} S\right)\right)}\right)$ is in $\mathcal{A}_{\mathbb{R}^{S}}$ hence $\mathcal{M}_{\left(C \mathbb{R}^{S}, \tau_{S}\left(O l_{1} S\right)\right)}$ is finer than the original structure on $C \mathbb{R}^{S}$. So $K C \mathbb{R}^{S} \subset K\left(C \mathbb{R}^{S}, \mathcal{M}_{\left(C \mathbb{R}^{S}, \tau_{S}\left(O l_{1} S\right)\right)}\right)$ and the desired inclusion follows from (5).

Let $\mathbf{T}^{\prime}=\left(T^{\prime}, \eta^{\prime}, \mu^{\prime}\right)$ be the monad of the adjunction $l_{1} \dashv O$ and let $\mathbf{T}=(T, \eta, \mu)$ be the monad of the adjunction $C^{\text {op }} \mathbb{R}^{-} \dashv D$.

If we show that the collection of mappings defined in (4) induces a monad morphism $\tau: \mathbf{T}^{\prime} \rightarrow \mathbf{T}$ then the assertion follows from the fact that $\mathbf{T C}$ is the category of Eilenberg-Moore algebras of $\mathbf{T}^{\prime}$ by categorical arguments [13].

We will use the notation $F$ for $C^{\text {op }} \mathbb{R}^{-}$.

1. $\tau: T^{\prime} \rightarrow T$ is a natural transformation. Take a map $f: S_{1} \longrightarrow S_{2}$ between sets. We have to verify the commutation of

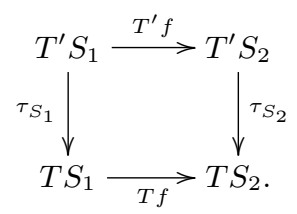

Let $b=\sum_{s \in S_{1}} b(s) \delta_{s} \in T^{\prime} S_{1}$. Then on the one hand we have that

$$
\begin{aligned}
\left(\tau_{S_{2}} \circ T^{\prime} f\right)(b) & =\tau_{S_{2}}\left(\sum_{s \in S_{1}} b(s) \delta_{f(s)}\right) \\
& =\sum_{s \in S_{1}} b(s) e v(-, f(s))
\end{aligned}
$$

and on the other hand we have

$$
\begin{aligned}
\left(T f \circ \tau_{S_{1}}\right)(b) & =\operatorname{DFf}\left(\sum_{s \in S_{1}} b(s) e v(-, s)\right) \\
& =\sum_{s \in S_{1}} b(s)(e v(-, s) \circ F f) \\
& =\sum_{s \in S_{1}} b(s) e v(-, f(s)) .
\end{aligned}
$$

2. $\tau$ rewrites the unit, i.e. we have to check that for an arbitrary set $S$ the diagram

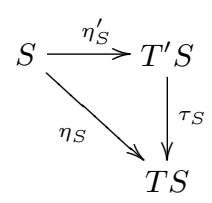

is commutative. But this is trivial: $\left(\tau_{S} \circ \eta_{S}^{\prime}\right)(s)=\tau_{S}\left(\delta_{s}\right)=\operatorname{ev}(-, s)=\eta_{S}(s)$ for all $s \in S$. 
3. Last we have to verify that $\tau$ rewrites multiplication, that is, for any set $S$ we have the commutation of the diagram

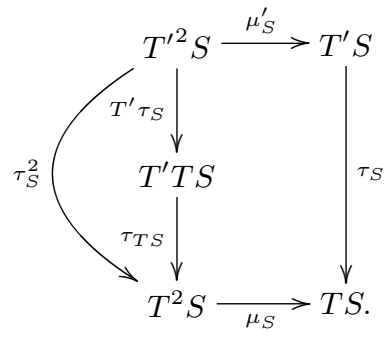

In order to show this, let $B \in T^{\prime 2} S$, where we write $B=\sum_{b \in T^{\prime} S} B(b) \delta_{b}$. Then $\mu_{S}^{\prime}(B)=$ $\sum_{b \in T^{\prime} S} B(b) b$, so

$$
\left(\tau_{S} \circ \mu_{S}^{\prime}\right)(B)=\sum_{s \in S} \sum_{b \in T^{\prime} S} B(b) b(s) \operatorname{ev}(-, s) .
$$

On the other hand we should compute $\left(\mu_{S} \circ \tau_{S}^{2}\right)(B)=\left(\mu_{S} \circ \tau_{T S} \circ T^{\prime} \tau_{S}\right)(B)$. First, note that

$$
T^{\prime} \tau_{S}(B)=\sum_{b \in T^{\prime} S} B(b) \delta_{\tau_{S}(b)},
$$

so

$$
\left(\tau_{T S} \circ T^{\prime} \tau_{S}\right)(B)=\sum_{b \in T^{\prime} S} B(b) \operatorname{ev}\left(-, \tau_{S}(b)\right)
$$

and

$$
\left(\mu_{S} \circ \tau_{T S} \circ T^{\prime} \tau_{S}\right)(B)=\sum_{b \in T^{\prime} S} B(b) \operatorname{ev}\left(-, \tau_{S}(b)\right) \circ \epsilon_{F S} .
$$

For all $a \in F S$ we have that

$$
\begin{aligned}
\operatorname{ev}\left(-, \tau_{S}(b)\right) \circ \epsilon_{F S}(a) & =\operatorname{ev}\left(\operatorname{ev}(-, a), \tau_{S}(b)\right) \\
& =\operatorname{ev}\left(\tau_{S}(b), a\right) \\
& =\tau_{S}(b)(a) \\
& =\left(\sum_{s \in S} b(s) \operatorname{ev}(-, s)\right)(a),
\end{aligned}
$$

hence

$$
\left(\mu_{S} \circ \tau_{T S} \circ T^{\prime} \tau_{S}\right)(B)=\sum_{b \in T^{\prime} S} B(b)\left(\sum_{s \in S} b(s) \operatorname{ev}(-, s)\right)=\sum_{b \in T^{\prime} S} \sum_{, s \in S} B(b) b(s) \operatorname{ev}(-, s) .
$$

Note that we have the commutative diagram

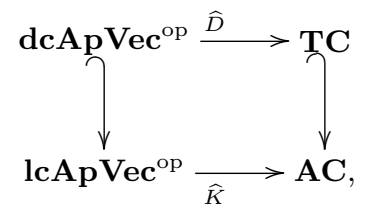

with $\widehat{K}$ the comparison functor of the adjunction $\mathbb{R}^{-} \dashv K[12]$.

Suppose that we have the following functors $F, G, H, I$ between the categories $\mathcal{A}, \mathcal{B}, \mathcal{C}$

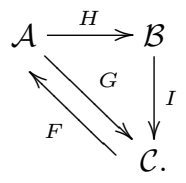


If $F$ is left adjoint to $G, I$ is full and faithful and $G=I \circ H$, then $F \circ I$ is left adjoint to $H$. In particular it follows from (6) and the fact that that $\widehat{K}$ has a left adjoint $W[12]$, that the composition $\mathbf{T C} \hookrightarrow \mathbf{A C} \stackrel{W}{\longrightarrow} \mathbf{l} \mathbf{c A p V e c}{ }^{\mathrm{op}} \stackrel{C^{\mathrm{op}}}{\longrightarrow} \mathbf{d} \mathbf{c} \mathbf{A} \mathbf{p V e c}{ }^{\mathrm{op}}$ is left adjoint to $\widehat{D}$.

We turn our attention to the connection with Banach spaces. It follows, from [6], that $L_{\widehat{K}} X$ is a Banach space if and only if $X$ is a dually complete space. Let

$$
L_{\widehat{D}}: \operatorname{dcApVec} \longrightarrow \mathrm{Ban}_{1}
$$

be the restriction, in domain and co-domain, of the functor $L_{\widehat{K}}$. Now, for a seminormed space $X$, the map

$$
X \rightarrow L_{\widehat{K}} \mathrm{E}_{\mathrm{pcu}} X: x \longmapsto \mathrm{ev}(-, x)
$$

is an isometrical bijection that is universal for $L_{\widehat{K}}$ [12]. So for a Banach space $X, L_{\mathrm{pcu}} X$ is dually complete and it follows that (7) is an isomorphism between Banach spaces which is universal for $L_{\widehat{D}}$. Thus the functor

$$
L_{\mathrm{pcu}}: \mathbf{B a n}_{1} \longrightarrow \mathbf{d c A p V e c}{ }^{\mathrm{op}}
$$

is left adjoint to $L_{\widehat{D}}$. It also follows that objects in the range of $L_{\widehat{D}}$ are isomorphism dense in the class of Banach spaces. In the sequel we put

$$
-^{(*)}:=C^{\mathrm{op}} L_{\mathrm{pcu}}: \mathbf{s N o r m} \mathbf{N o d}_{1} \rightarrow \mathbf{d c A p V e c}{ }^{\mathrm{op}} .
$$

Theorem 2.3. The functor $L_{\widehat{D}^{-}}{ }^{(*)}$ is left adjoint to the embedding $\mathbf{B a n}_{1} \hookrightarrow \mathbf{s N o r m}_{1}$.

Proof. From composition of adjunctions it follows that $-^{(*)}$ is left adjoint to the composition $\mathbf{d c A p V e c}^{\mathrm{op}} \hookrightarrow \mathbf{l c A p V e c}^{\mathrm{op}} \stackrel{L_{\widehat{K}}}{\longrightarrow} \mathbf{s N o r m}_{1}$. The corresponding unit on a seminormed space $X$ is given by $\eta_{X}: X \rightarrow L_{\widehat{K}} X^{(*)}=L_{\widehat{D}} X^{(*)}: x \longmapsto \operatorname{ev}(-, x)$. We will show that this is a universal map with respect to the class of Banach spaces.

Let $Y$ be a Banach space and $f: X \rightarrow Y$ be a linear non-expansive map. Let $f^{(*)}: Y^{(*)} \rightarrow X^{(*)}$ be the unique dcApVec-morphism satisfying $\eta_{Y} \circ f=L_{\widehat{K}} f^{(*)} \circ \eta_{X}=L_{\widehat{D}} f^{(*)} \circ \eta_{X}$. Note that $\eta_{Y}$ is an isomorphism of Banach spaces, so there exists a linear non-expansive map $g: L_{\widehat{D}} X^{(*)} \rightarrow Y$ such that $f=g \circ \eta_{X}$. Any such $g$ is uniquely determined on the closure of $\eta_{X}(X)$ in $L_{\widehat{D}} X^{(*)}$. Let $\widetilde{X}$ denote this sub Banach space. We are finished if we show that $\widetilde{X}=L_{\widehat{D}} X^{(*)}$.

Let $X^{*}$ be the algebraic dual of $X$. For any $x \in O X$ we have that $\operatorname{ev}(-, x) \in \tilde{X}$. Moreover, since $\|\operatorname{ev}(-, x)\| \leq\|x\|$ we then also have that $\operatorname{ev}(-, x) \in O(\widetilde{X})$. So we see that the map id : $\left(X^{*}, \mathcal{M}_{\left(X^{*}, O(\widetilde{X})\right)}\right) \longrightarrow L_{\mathrm{pcu}} X$ is a contraction. Since it follows from the formula (1) that $\left(X^{*}, \mathcal{M}_{\left(X^{*}, O(\widetilde{X})\right)}\right)$ is dually complete, the injection $\left(X^{*}, \mathcal{M}_{\left(X^{*}, O(\widetilde{X})\right)}\right) \hookrightarrow C L_{\mathrm{pcu}} X=X^{(*)}$ is a contraction. This embedding is necessarily the identity map, so $\widetilde{X}=L_{\widehat{D}}\left(X^{*}, \mathcal{M}_{\left(X^{*}, O(\widetilde{X})\right)}\right)=L_{\widehat{D}} X^{(*)}$.

Corollary 2.4. Let $X$ be a normed space. Then the embedding $X \hookrightarrow L_{\widehat{D}} X^{(*)}: x \longmapsto \operatorname{ev}(-, x)$ is the completion.

\section{References}

[1] D. Cioranescu and P. Donato. An Introduction to Homogenization. Oxford lecture series in mathematics and its applications. Oxford University Press, 1999.

[2] R. Lowen. Approach spaces: The Missing Link in the Topology-Uniformity-Metric Triad. Oxford Mathematical Monographs. Oxford University Press, 1997.

[3] R. Lowen and M. Sioen. Approximations in functional analysis. Results in Mathematics, 37:345$372,2000$.

[4] R. Lowen and S. Verwulgen. Approach vector spaces. Houston Journal of Mathematics, 30(4):1127$1142,2004$.

[5] D. Pumplün. Absolutely convex modules and Saks spaces. Journal of Pure and Applied Algebra, 155:257-270, 2001. 
[6] D. Pumplün and H. Röhrl. Banach spaces and totally convex spaces i. Communications in Algebra, 12(8):953-1019, 1984.

[7] D. Pumplün and H. Röhrl. Banach spaces and totally convex spaces ii. Communications in Algebra, 13(5):1047-1113, 1985.

[8] D. Pumplün and H. Röhrl. Separated totally convex spaces. Manusc. Math, 50:145-183, 1985.

[9] D. Pumplün and H. Röhrl. The coproduct of totally convex spaces. Beitr. Alg. Geom., 24:249-278, 1987.

[10] D. Pumplün and H. Röhrl. Convexity theories V: Extensions of absolutely convex modules. Applied Categorical Structures, 8:527-543, 2000.

[11] Z. Semadeni. Monads and their Eilenberg-Moore algebras in functional analysis. Queen's papers in pure and applied mathematics, 33:1-98, 1973.

[12] M. Sioen and S. Verwulgen. On the absolute convex structure of linear contractions. submitted.

[13] M. Sioen and S. Verwulgen. On the algebraic structure of dual unit balls of seminormed spaces. submitted.

[14] M. Sioen and S. Verwulgen. Locally convex approach spaces. Applied General Topology, 4(2):263279, 2003.

[15] M. Sioen and S. Verwulgen. Quantified functional analysis: recapturing the dual unit ball. Results in Mathematics, 45(3-4):359-369, 2004.

[16] S. Verwulgen. Dual adjunctions in functional analysis: quantified versus qualitative theory. submitted. 